\title{
Puérperas de sífilis congênita de uma maternidade de Cabo Frio-RJ: levantamento do perfil epidemiológico
}

RESUMO | Objetivo: identificar o perfil epidemiológico de puérperas de sífilis congênita internadas em uma maternidade do município de Cabo Frio-RJ. O cenário foi uma maternidade do município de Cabo Frio. Os sujeitos foram 24 puérperas de recém-nascidos com sífilis congênita. A maior parte das entrevistadas tem entre 18 e 24 anos (66,7\%), apresentam o ensino médio completo (54,2\%), são solteiras (75\%), declaram-se negras ( $54,2 \%$ ) e possuem renda familiar de um salário mínimo (45,8\%). A maioria não tem parceiro fixo $(66,7 \%)$ e não utiliza preservativo $(50 \%)$ durante as relações sexuais. O maior número declarou ter realizado o pré-natal $(75 \%)$, tendo o diagnóstico de sífilis entre 3 a 6 meses de gestação (54,2\%). Majoritariamente o parceiro não recebeu tratamento $(62,5 \%)$ e não houve orientação de enfermagem no pré-natal $(62,5 \%)$. A redução da sífilis na gestação e consequentemente da sífilis congênita, está relacionada a um atendimento prioritário e adequado na rede básica de saúde.

Palavras-chaves: Sifilis Congênita; Transmissão Vertical de Doença Infecciosa; Saúde Materno-Infantil.

ABSTRACT | Objective: identify the epidemiological profile of postpartum women with congenital syphilis admitted to a maternity hospital in Cabo Frio-RJ. The scenario was a maternity hospital in Cabo Frio. The subjects were 24 puerperal women with congenital syphilis. Most of the interviewees are between 18 and 24 years old (66.7\%), have completed high school (54.2\%), are single (75\%), declare themselves black (54.2\%) and have income minimum wage (45.8\%). Most have no steady partner (66.7\%) and do not use condoms (50\%) during sexual intercourse. The largest number reported having had prenatal care (75\%), with the diagnosis of syphilis between 3 and 6 months of gestation (54.2\%). Mostly the partner did not receive treatment (62.5\%) and there was no prenatal nursing guidance (62.5\%). The reduction of syphilis in pregnancy and consequently congenital syphilis is related to priority and adequate care in the basic health network.

Keywords: Congenital Syphilis; Vertical Transmission of Infectious Doença; Maternal and Child Health.

RESUMEN I Objetivo: identificar el perfil epidemiológico de mujeres posparto con sífilis congénita ingresadas en un hospital de maternidad en Cabo Frio-RJ. El escenario era un hospital de maternidad en Cabo Frio. Los sujetos fueron 24 mujeres puerperales con sífilis congénita. La mayoría de los entrevistados tienen entre 18 y 24 años (66.7\%), han completado la escuela secundaria (54.2\%), son solteros (75\%), se declaran negros (54.2\%) y tienen ingresos salario mínimo (45.8\%). La mayoría no tiene pareja estable (66.7\%) y no usa condones (50\%) durante las relaciones sexuales. El mayor número informó haber recibido atención prenatal (75\%), con el diagnóstico de sífilis entre los 3 y 6 meses de gestación (54,2\%). En su mayoría, la pareja no recibió tratamiento $(62.5 \%)$ y no hubo orientación de enfermería prenatal (62.5\%). La reducción de la sífilis en el embarazo y, en consecuencia, la sífilis congénita está relacionada con la atención prioritaria y adecuada en la red básica de salud.

Descriptores: Sífilis Congénita; Transmisión Vertical de Enfermedades Infecciosas; Salud Materna e Infantil.

\section{Cristiane Nascimento Felipe}

Enfermeira pela Universidade Veiga de Almeida.

\section{Daniela da Silva Freitas}

Enfermeira pela Universidade Veiga de Almeida.

\section{Luciana da Costa Nogueira Cerqueira}

Enfermeira. Mestre em biociência pela Universidade Federal do Rio de Janeiro. Professora do curso de graduação em Enfermagem da Universidade Veiga de Almeida.

\section{Priscila Pradonoff Oliveira}

Enfermeira. Mestre em psicanálise, saúde e sociedade pela Universidade Veiga de Almeida. Professora do curso de graduação em Enfermagem da Universidade Veiga de Almeida.

\section{Carlos Eduardo Peres Sampaio}

Professor titular da Universidade Veiga de Almeida. Professor associado do departamento de enfermagem médico cirúrgica da Universidade do Estado do Rio de Janeiro.

\section{Giselle Barcellos Oliveira Koeppe}

Enfermeira. Mestre e Doutora em Enfermagem pela Escola de Enfermagem Anna Nery/Universidade Federal do Rio de Janeiro. Professora do curso de graduação em Enfermagem da Universidade Veiga de Almeida.

Recebido em: 28/05/2019

Aprovado em: : 28/05/2019 


\section{INTRODUÇÃO}

A sífilis é uma Infecção Sexualmente Transmissível (IST) causada pela espiroqueta treponema pallidum que, apesar de apresentar diagnóstico e tratamento bem estabelecidos e de baixo custo, é considerada um importante agravo de saúde pública. Quando não tratada precocemente, esta enfermidade pode se transformar em uma condição crônica com sequelas irreversíveis a longo prazo. Uma das maiores preocupações do controle desta doença é a infecção de muIheres em idade reprodutiva, que pode acarretar a ocorrência de sífilis congênita por meio da transmissão vertical ${ }^{(1,2)}$.

A sífilis congênita representa uma infecção transmitida predominantemente por via transplacentária em qualquer momento da gestação ou estágio clínico da doença em gestante não tratada, ou inadequadamente tratada. Pode resultar em consequências graves, como óbito infantil, aborto e natimortalidade, além de sequelas como deficiência visual, auditiva, física e mental, que acarretam prejuízo no desenvolvimento dessas crianças. Contudo, a transmissão vertical da sífilis é absolutamente evitável, desde que a gestante seja diagnosticada e tratada de forma acertada e precisa. As ações preventivas estão disponíveis no pré-natal da gestante com rastreamento sorológico para a sífilis e o tratamento oportuno da infecção quando diagnosticada, de forma mais precoce possível ${ }^{(2)}$

A sífilis congênita é responsável por altas taxas de morbidade e mortalidade, podendo chegar a $40 \%$ a taxa de abortamento, óbito fetal e morte neonatal dos conceptos infectados a partir de mães com sífilis não tratadas ${ }^{(1,2)}$. Esta infecção atinge um milhão de gestantes por ano em todo o mundo, levando a mais de 300 mil mortes fetais e neonatais e colocando em risco de morte prematura mais de 200 mil crianças. Na América Latina e Caribe, estima-se que entre 166.000 e 344.000 recém-nascidos nasçam com sífilis congênita anualmente. Especificamente no
Brasil, o número de casos de sífilis em gestantes, congênita e adquirida, tem apresentado um aumento constante nos últimos anos ${ }^{(3)}$.

A sífilis congênita tornou-se de notificação compulsória a partir da Portaria n. ${ }^{\circ}$ 542/1986 do Ministério da Saúde ${ }^{(4)}$, porém a subnotificação ainda é uma realidade amplamente vista, assim como o crescente aumento dos casos entre a população, apontando para uma deficiência no manejo deste problema de saúde. Embora o controle desta infecção represente uma meta primordial para o sistema de saúde, em várias regiões do Brasil ainda se verifica a dificuldade em atingir tal objetivo. Tal situação sugere a ocorrência de um pré-natal insatisfatório, do ponto de vista do diagnóstico e tratamento desta doença ${ }^{(5)}$.

Os dados alarmantes de casos de sífilis congênita trazem à reflexão a importância do conhecimento sobre esta temática, enfatizando-se o destaque para as investigações epidemiológicas, que permitem direcionar ações de saúde com base no perfil da população assistida. Isso porque os achados epidemiológicos apontam indicativos importantes para uma possível reorganização dos serviços de saúde, seja a nível estrutural, organizacional ou voltado, especificamente, para as questões inerentes ao diagnóstico e ao tratamento da população.

Neste contexto, e diante dos apontamentos trazidos aqui, emergiu o interesse de levantar o perfil das puérperas de sífilis congênita, buscando desvelar mais sobre o acometimento da população por esta doença.

O reconhecimento do perfil da cliente exposta à sífilis congênita em determinado município, frente às repercussões de morbimortalidade desta doença, é de suma importância para a identificação dos desafios ainda existentes no controle de sua transmissão ${ }^{(6)}$.

Com base nos apontamentos trazidos até aqui, emergiu a seguinte questão norteadora: Qual o perfil epidemiológico de puérperas de sífilis congênita internadas em uma maternidade do município de Cabo Frio-RJ? Dessa forma, o objetivo traçado para este estudo foi identificar o perfil epidemiológico de puérperas de sífilis congênita internadas em uma maternidade do município de Cabo Frio-RJ.

\section{METODOLOGIA}

Trata-se de um estudo descritivo, exploratório, com abordagem quantitativa.

O cenário do estudo foi uma maternidade, localizada no município de Cabo Frio, no Estado do Rio de Janeiro. A escolha por este local se deu por se tratar de um serviço de referência para parto na região.

Os sujeitos da pesquisa foram 24 puérperas internadas na instituição no período da coleta de dados, que atenderam aos seguintes critérios de inclusão: mães de recém-nascidos que tiveram diagnóstico de sífilis congênita ao nascimento; maiores de 18 anos; estáveis hemodinamicamente; e que aceitaram prontamente a participar da pesquisa. As participantes foram convidadas a participarem da pesquisa, sendo informadas sobre os objetivos, tendo sua concordância em participar mediante assinatura no Termo de Consentimento Livre e Esclarecido (TCLE), sendo garantida a preservação das identidades das puérperas.

A coleta de dados se deu a partir de um questionário com questões fechadas, elaborado para este fim. A investigação ocorreu nos meses de outubro e novembro de 2018, sendo contempladas todas as mulheres internadas no período que atenderam aos critérios de inclusão do estudo.

Os dados coletados foram tabulados em tabelas e gráficos analíticos, o que proporcionou uma análise estatística descritiva.

O desenvolvimento da pesquisa foi autorizado pela diretoria geral da instituição, mediante assinatura em carta de anuência. $\mathrm{O}$ estudo respeitou os aspectos éticos previstos na Resolução n. ${ }^{\circ} 466$ de 12 de dezembro de 2012, do Conselho Nacional de Saúde - CNS(7), sendo avaliado pelo Comitê de Ética em Pesquisa da Universidade Veiga de Almeida (CEP/ UVA), pelo Parecer Consubstanciado n. ${ }^{\circ}$ 2.907.088 de 20 de setembro de 2018. 


\section{RESULTADOS}

A faixa etária materna mais frequente foi de 18 a 24 anos $(66,7 \%)$. A maior parte das participantes apresenta

o ensino médio completo (54,2\%), são solteiras $(75 \%)$ e declaram-se negras
(54,2\%). A renda familiar de um salário mínimo corresponde a $45,8 \%$ das entrevistadas (Tabela 1).

\section{Tabela 1. Distribuição das características demográficas de puérperas de sifilis congênita internadas em uma maternidade no} municipio de Cabo Frio-RJ. Brasil, 2018.

\section{CARACTERÍSTICAS DEMOGRÁFICAS}

Faixa etária

$\begin{array}{cccc}18 \text { 24 anos } & 16 & 66,7 & 29,1 \\ 24 \text { 30 anos } & 7 & 4,2 \\ \geq 30 \text { anos } & 1 & 100\end{array}$

Escolaridade

\begin{tabular}{|c|c|c|}
\hline Fundamental incompleto & 2 & 8,3 \\
\hline Fundamental completo & 9 & 37,5 \\
\hline Médio completo & 13 & 54,2 \\
\hline \multicolumn{3}{|l|}{ Estado civil } \\
\hline Solteira & 13 & 54,2 \\
\hline Divorciada & 4 & 16,7 \\
\hline Total & 24 & 100 \\
\hline \multicolumn{3}{|l|}{ Cor ou raça } \\
\hline Negra & 13 & 54,2 \\
\hline \multicolumn{3}{|l|}{ Renda familiar } \\
\hline 1 salário mínimo & 11 & 45,8 \\
\hline < 1 salário mínimo & 7 & 29,1 \\
\hline >1 salário mínimo & 5 & 20,9 \\
\hline Não informado & 1 & 4,2 \\
\hline Total & 24 & 100 \\
\hline
\end{tabular}

A maior parte das puérperas $(66,7 \%)$ apontou ter um parceiro fixo e não fazer uso de preservativo (50\%) durante as relações sexuais (Tabela 2).
O maior número (75\%) das participantes declarou ter realizado o pré-natal,

Tabela 2. Distribuição das características relacionadas ao comportamento frente às relações sexuais de puérperas de sifflis congênita internadas em uma maternidade no município de Cabo Frio-RJ. Brasil, 2018.

\section{CARACTERÍSTICAS DO COMPORTAMENTO} SEXUAL

N
$\%$

Presença de parceiro fixo 


\begin{tabular}{|ccc|}
\hline Sim & 16 & 66,7 \\
\hline Não & 8 & 33,3 \\
\hline Uso de preservativo & 12 & 50 \\
\hline Não & 7 & 29,1 \\
\hline Sempre & 7 & 20,9 \\
\hline Às vezes & 5 & 100 \\
\hline Total & 24 & 24 \\
\hline
\end{tabular}

sendo o diagnóstico de sífilis constatado maior parte das vezes $(62,5 \%)$ o parceiro de 3 a 6 meses de gestação (54,2\%). Na não recebeu tratamento e 62,5\% delas indicou que recebeu orientação de enfermagem durante o pré-natal (Tabela 3).

Tabela 3. Distribuição das características da assistência pré-natal de puérperas de sifilis congênita internadas em uma maternidade no municipio de Cabo Frio-RJ. Brasil, 2018.

\section{CARACTERÍSTICAS DA ASSISTÊNCIA NO} PRÉ-NATAL

Realizou pré-natal

Sim

Total
$\mathrm{N}$

18

6

24
$\%$

75

25

100

\section{Período do diagnóstico da sífilis na} gestação

$\begin{array}{ccc}03 \text { meses } & 6 & 25 \\ 36 \text { meses } & 13 & 54,2 \\ >6 \text { meses } & 4 & 16,7 \\ \text { Após o parto } & 1 & 4,2 \\ \text { Total } & 24 & 100\end{array}$

\section{Tratamento parceiro}

$\begin{array}{ccc}\text { Sim } & 9 & 37,5 \\ \text { Não } & 15 & 62,5 \\ \text { Total } & 24 & 100\end{array}$

Orientações de enfermagem durante 0 pré-natal

$\begin{array}{ccc}\text { Sim } & 15 & 62,5 \\ \text { Não } & 9 & 37,5 \\ \text { Total } & 24 & 100\end{array}$




\section{DISCUSSÃO}

Este estudo apontou um perfil demográfico semelhante ao encontrado em outras pesquisas realizadas ${ }^{(5-6,8-12)}$. Fica notório que esta população se enquadra em um padrão caracterizado por mulheres jovens, não brancas, de baixa escolaridade e solteiras.

Quanto ao predomínio de mulheres jovens nesta clientela, pode-se dizer que, apesar da sífilis congênita não ser considerada uma doença que tenha preferência por grupos populacionais específicos, mulheres novas estão mais predispostas a adquirirem a infecção em decorrência de seu comportamento frente às relações sexuais ${ }^{(6)}$.

O baixo poder aquisitivo verificado nesta pesquisa reafirma a realidade de muitos usuários do Sistema Único de Saúde (SUS). Neste contexto, evidências indicam que a pobreza e suas subsequentes condições vulnerabilizantes, dentre as quais destaca-se a dificuldade de acesso e qualidade da assistência pré-natal ofertada nos serviços de saúde, estão consideravelmente atreladas à ocorrência de sífilis entre as gestantes ${ }^{(13)}$.

A partir do comportamento frente às relações sexuais das participantes do estudo, pode-se inferir que a presença de parceiro fixo não inviabiliza a ocorrência de IST. Tal relação está intimamente ligada ao fato de que muitas mulheres acreditam que uma união estável lhes garante a autopreservação deste tipo de infecção. Esta percepção equivocada pode ser uma das razões que levam as puérperas abordadas na pesquisa a não se apropriarem de forma adequada do preservativo durante suas relações sexuais, conforme apontam os dados.

Neste contexto, destaca-se a importância da realização de ações de educação em saúde voltadas para o incentivo das práticas sexuais seguras com incorporação do uso do preserva-
66

enfermeiro que atua em prénatal representa a peça chave na prevenção e detecção das sífilis gestacional e congênita. Dentre suas funções está a educação em saúde, que funciona como ferramenta indispensável para o autocuidado da gestante, favorecendo melhoria na qualidade de vida do binômio mãefilho. tivo, não apenas como método contraceptivo, mas também para prevenir a ocorrência de ISTs ${ }^{(14)}$.

Fatores associados à prevenção das ISTs devem ser foco no estabelecimento de políticas de prevenção. No Brasil, são inúmeras as medidas nacionais, estaduais e municipais que favorecem a ampliação do acesso universal e gratuito de preservativos, com o intuito de ampliar a prática da relação sexual protegida. Neste universo, deve haver uma ampla acessibilidade aos serviços de saúde para diagnóstico e tratamento precoce, evitando complicações decorrentes desse tipo de infecção. Além disso, a educação permanente de profissionais necessita ser largamente propagada, levando-se em consideração as particularidades e as diferentes situações de vulnerabilidade da população ${ }^{(15)}$.

Conforme aponta os dados do estudo, a maior parte das participantes declarou ter realizado o pré-natal de forma preconizada. Ainda assim, foi verificado que a maioria não recebeu o diagnóstico da sífilis antes do primeiro trimestre, assim como não foi realizado o tratamento de seus parceiros. Tais informações são constatadas também em outras pesquisas, que mostraram o tratamento inadequado da sífilis na gestante e em seu parceiro, entre mulheres que haviam realizado o pré-natal ${ }^{(5,6,10,11)}$.

O controle da sífilis congênita está atrelado ao diagnóstico precoce da gestante associado ao seu tratamento imediato, além de ser fundamental tratar concomitantemente seu parceiro, a fim de diminuir ou evitar reinfecções ${ }^{(6,14)}$. O pré-natal representa o mecanismo mais importante para que esta detecção e prevenção ocorram de forma satisfatória, devendo, portanto, ser realizado de acordo com as normas preconizadas, que visam garantir o bem-estar materno-infantil.

A falha na detecção e tratamento da sífilis na gestação, resultando na 
ocorrência da sífilis congênita, aponta falhas na classificação desta condição durante o pré-natal. Isso atesta a necessidade da melhoria da assistência na atenção básica, seja nas ações relacionadas à educação em saúde, seja no rigor estabelecido no diagnóstico e tratamento da infecção ${ }^{(11,14)}$.

Ainda neste contexto, vale destacar que o maior número das gestantes afirmou ter recebido orientações da enfermagem durante o pré-natal, o que remete a uma preocupação acerca das atividades do enfermeiro nesta modalidade de atenção. Este profissional precisa desempenhar seu papel de educador junto às gestantes e no treinamento de sua equipe, com vistas a promover o manejo adequado da sífilis, evitando sequelas indesejadas.

O enfermeiro que atua em pré-natal representa a peça chave na prevenção e detecção das sífilis gestacional e congênita. Dentre suas funções está a educação em saúde, que funciona como ferramenta indispensável para o autocuidado da gestante, favorecen- do melhoria na qualidade de vida do binômio mãe-filho. Para tanto, é primordial que o enfermeiro, frente aos casos de sífilis gestacional, não aja apenas como cumpridor de tarefas técnicas e assistenciais, mas que coloque em prática suas competências de forma efetiva, fornecendo um pré-natal completo e de qualidade ${ }^{(16)}$.

\section{CONCLUSÃO}

Foi possível observar que as características demográficas apresentadas são semelhantes àquelas observadas em estudos anteriores. Isso é de grande valia para o enriquecimento de estudos epidemiológicos, que permitem a elaboração de estratégias direcionadas à população.

A pesquisa mostrou alguns problemas existentes relacionados ao diagnóstico precoce e tratamento adequado em gestantes e recém-nascidos. Este dado sugere que a qualidade do pré-natal recebido pela gestante não é suficiente para garantir o controle da sífilis congênita, contrariando as diretrizes definidas pelo Ministério da Saúde do Brasil para a redução desta condição de saúde entre a população.

A redução da ocorrência de sífilis na gestação e, consequentemente, da sífilis congênita, está intimamente relacionada a um atendimento prioritário na rede básica de saúde, em que sejam incluídas orientações de educação sexual mais efetivas, acompanhamento pré-natal eficaz e busca ativa de cuidado e atenção à população circunscrita. Tais atitudes são representadas pela adoção de medidas mais efetivas de prevenção sistematicamente aplicadas à mulher nos serviços de saúde.

Nesse contexto, o enfermeiro está inserido exercendo sua função nata de educador, oferecendo orientações pertinentes e precisas à clientela atendida. Além disso, este profissional deve participar da elaboração das estratégias que alcancem a diminuição dos índices e prevalência da sífilis congênita.

\section{Referências}

1. Ministério da Saúde (BR). Secretaria de Vigilância em Saúde. Departamento de Vigilância, Prevenção e Controle das Doenças Sexualmente Transmissiveis, Aids e Hepatites Virais. Manual Técnico para Diagnóstico da Sífilis. Brasília: Ministério da Saúde; 2016.

2. Secretaria de Estado da Saúde de São Paulo. Centro de Controle de Doenças. Programa Estadual de DST/Aids. Centro de Referência e Treinamento DST/Aids. Guia de bolso para o manejo da sífilis em gestantes e sífilis congênita. São Paulo: Secretaria de Estado da Saúde; 2016.

3. Ministério da Saúde (BR). Secretaria de Vigilância em Saúde. Boletim epidemiológico sífilis 2017; 48(36).

4. Ministério da Saúde (BR). Portaria n. ${ }^{\circ}$ 542, de 22 de dezembro de 1986. Inclui na relação de doenças de notificação compulsória no território nacional (Portaria Ministerial n. ${ }^{\circ} 608$, de 28 de outubro de 1979) a sífilis congênita e a aids. Diário Oficial da República Federativa do Brasil. 1986 dez. 24; Seção 1. p.19827. 5. Lafetá KRG, Martelli Júnior $H$, Silveira MF, Paranaíba LMR. Sífilis materna e congênita, subnotificação e difícil controle. Revista BRAS epidemiol. 2016; 19(1): 63-74.

6. Moreira KFA, Oliveira DM, Alencar LN, Cavalcante DFB, Pinheiro AS, Orfão NH. Perfil dos casos notificados de sífilis congênita. Revista Cogitare Enferm. 2017; (22)2: e48949.

7. Conselho Nacional de Saúde (BR). Resolução n. ${ }^{\circ} 466$, de 12 de dezembro de 2012. Aprova diretrizes e normas regulamentadoras de pesquisas envolvendo seres humanos. Diário Oficial da União 13 jun 2013; Seção 1.

8. Domingues RMSM, Leal MC. Incidência de sífilis congênita e fatores associados à transmissão vertical da sífilis: dados do estudo Nascer no Brasil. Cad.
Saúde pública. 2016; 32(6):e00082415.

9. Chinazzo LK, Leon CA. Perfil clínico e epidemiológico da sífilis congênita na unidade de internação de um hospital universitário. Boletim científico de pediatria. 2015; 4(3); 65-69.

10. Silva IMD, Leal EMM, Pacheco HF, Souza Júnior JG, Silva FS. Perfil epidemiológico da sífilis congênita. Rev. Enferm. UFPE. 2019; 13(3): 604-13.

11. França ISX, Batista JDL, Coura AS, Oliveira CF, Araújo AKF, Sousa FS. Fatores associados à notificação da sífilis congênita: um indicador de qualidade da assistência pré-natal. Revista Rene. 2015; 16(3): 374-81.

12. Nonato SM, Melo APS, Guimarães MDC. Sífilis na gestação e fatores associados à sífilis congênita em Belo Horizonte-MG, 2010-2013. Epidemiol. Serv. Saúde. 2015; 24(4): 681-694.

13. Macêdo VC, Lira PIC, Frias PG, Romaguera LMD, Caires SF, Ximenes RAA. Fatores de risco para sífilis em mulheres: estudo caso-controle. Rev Saúde Pública. 2017; 51:78.

14. Signor M, Spagnolo LML, Tomberg JO, Gobatto M, Stofel NS. Distribuição especial e caracterização de casos de sífilis congênita. Rev. Enferm. UFPE. 2018; 12(2): 398-406.

15. Pinto VM, Basso CR, Barros CRS, Gutierrez EB. Fatores associados às infecções sexualmente transmissíveis: inquérito populacional de São Paulo, Brasil. Ciência \& saúde coletiva. 2018; 23(7): 2423-32.

16. Araújo AM, Fidélis EPB, Silva ASD, Lira LBS, Gomes WQ, Pessoa IR, Viana MER. Atuação do enfermeiro na assistência ao pré-natal versus sífilis: uma revisão integrativa. Interfaces científicas-saúde e ambiente. 2018; 6(2): 95-110. 